\title{
RELAÇÃO ENTRE ATIVIDADE FÍSICA E DEPRESSÃO EM IDOSOS: UMA REVISÃO INTEGRATIVA
}

\author{
RELATIONSHIP BETWEEN PHYSICAL ACTIVITY AND DEPRESSION IN ELDERLY: \\ AN INTEGRATING REVIEW
}

\section{Gabriel Augusto Bernardes Mendes ${ }^{a *}$, Maria Virgínia de Carvalho ${ }^{b * *}$, Antonio Márcio Teodoro Cordeiro Silvac*, Rogério José de Almeida ${ }^{\mathrm{d} *}$}

\author{
agabriel_augustobmendes@hotmail.com, bmvcarvalho20@hotmail.com, cmarciocmed@gmail.com, drogeriopucgo@gmail.com \\ *Pontifícia Universidade Católica de Goiás - Goiânia (GO), Brasil
}

**Universidade Federal de Goiás - Goiânia (GO), Brasil

Data de recebimento de artigo: 03/05/2017

Data de aceite do artigo: 05/10/2017

\section{RESUMO}

Introduçáo: Evidências científicas vêm demonstrando que o envelhecimento populacional é uma realidade. Os idosos estão cada vez mais saudáveis, produtivos e praticantes de atividade física. Entretanto, um dos agravos mais impactantes nessa populaçáo é a depressão. Objetivo: Este artigo tem por objetivo analisar em uma perspectiva biopsicossocial a relação entre a prática da atividade física e a depressão nos idosos. Métodos: Trata-se de uma revisão integrativa da literatura científica acerca da relação entre atividade física e depressão em idosos. Para a seleção dos artigos científicos foram consultadas as bases de dados da Biblioteca Virtual em Saúde (BVS), dos Periódicos Capes e do PubMed. Resultados: Emergiram e foram analisadas três categorias do fenômeno: relação entre atividade física e depressão em idosos; fatores biológicos da atividade física e depressão em idosos; atividade física em grupo e a depressão em idosos. Conclusáo: Foram identificadas evidências de que a prática regular de atividade física pode melhorar o condicionamento físico, a regulação hormonal e é alternativa eficaz para convívio em grupos sociais. Conclui-se que a prática regular de exercícios físicos em pessoas idosas tem papel fundamental na prevenção e diminuição dos sintomas depressivos.

Palavras-chave: Atividade física; depressão; idoso; revisão integrativa.

\section{ABSTRACT}

Introduction: Scientific evidence has shown that population ageing is a reality. The elderly is becoming healthier, productive and practicing physical activity. However, one of the most striking injuries in this population is depression. Objective: This article aims to analyze in a biopsychosocial perspective the relationship between the practice of physical activity and depression in the elderly. Method: This is an integrative review of the scientific literature concerning the relationship between physical activity and depression in the elderly. For the selection of scientific articles, the databases Virtual Health Library, Capes Periodicals and PubMed were consulted. Results: Three categories of the phenomenon emerged and were analyzed: relationship between physical activity and depression in the elderly, biological factors of physical activity and depression in the elderly and group physical activity and depression in the elderly. Conclusion: Evidence has been found that the regular practice of physical activity can improve physical conditioning, hormonal regulation and is an effective alternative for social group living. It is concluded that regular physical exercise in the elderly plays a fundamental role in the prevention and reduction of depressive symptoms.

Keywords: Physical activity; depression; elderly; integrative review. 


\section{Introdução}

O envelhecimento populacional refere-se à uma mudança na estrutura etária da população, tal fato vem ocasionando um aumento da quantidade de pessoas idosas no Brasil e no mundo ${ }^{1}$. Estima-se um aumento de $250 \%$ nos países em desenvolvimento e de $71 \%$ nos países desenvolvidos entre os anos de 2010 e $2050^{2}$. No Brasil, os idosos já representam cerca de $10 \%$ da população geral e estima-se que, no ano de 2050, 15\% da população brasileira terá 70 anos ou mais ${ }^{3}$.

Evidências já demonstraram que os novos idosos brasileiros estão mais saudáveis, vivem mais e são mais produtivos do que os do passado ${ }^{2-4}$. Há um envelhecimento ativo da população, fato esse que confirma a ideia de que quanto mais ativo ou quanto maior a prática de exercício físico pelo idoso, melhor é seu estado de saúde, a satisfação com a vida e, consequentemente, melhor é a qualidade de vida ${ }^{2,4}$.

Nessa fase da vida, um importante agravo à saúde do idoso é a depressão, uma doença psiquiátrica recorrente e incapacitante ${ }^{5}$, caracterizada pela perda de prazer ou interesse em atividades do cotidiano e humor deprimido. Distúrbios depressivos, isolados ou em combinaçáo com outras condiçóes, são os transtornos mais prevalentes em idosos, que muitas vezes náo apresentam resposta satisfatória aos tratamentos medicamentosos em comparação com os indivíduos mais jovens ${ }^{6}$.

Há uma necessidade de investigaçóes mais abrangentes dos estados depressivos nessa população ${ }^{7}$. Destacam-se, nesse ponto, os tratamentos não medicamentosos, como a realização de atividade física regular. $\mathrm{O}$ incentivo ao aumento da prática de atividade física na população em geral é uma estratégia promissora no avanço da saúde pública, com foco nas doenças crônicas, reduzindo a morbidade e mortalidade decorrentes dessas doenças ${ }^{8}$.

A interação entre aspectos sociais, físicos e mentais, a independência econômica e nas atividades diárias, a integração social e o suporte familiar - que otimizam oportunidades de saúde e segurança - implicam a perspectiva de um envelhecimento ativo e saudável. Observando isso, o papel da atividade física para a promoção da saúde mental do idoso se torna fundamental?.

Partiu-se, portanto, do interesse em buscar na literatura científica, por meio de uma revisão integrativa, evidências em pesquisas que auxiliaram na discussão do seguinte questionamento: Qual a relação entre atividade física e a depressão na população idosa? Assim, este estudo teve por objetivo analisar em uma perspectiva biopsicossocial a relação entre a prática da atividade física e a depressão nos idosos.

\section{Métodos}

Este estudo trata-se de uma revisão integrativa da literatura científica acerca da relação entre atividade física e depressáo em idosos. Para responder ao questionamento proposto optou-se por esse tipo de revisão de literatura, já que visa realizar uma síntese de múltiplas conclusóes científicas. Esta é uma pesquisa realizada de maneira sistemática e ordenada, com base no referencial teórico da prática baseada em evidências ${ }^{10}$, e envolveu a definição de um problema, a busca e análise crítica das evidências disponíveis e a avaliação integrativa dos resultados obtidos.

Para a seleção dos artigos científicos, foram consultadas as bases de dados na Biblioteca Virtual em Saúde (BVS), os Periódicos Capes e o PubMed. Para a busca eletrônica dos artigos indexados nas bases de dados, foram utilizados os seguintes Descritores das Ciências da Saúde (DeCS) e suas combinações nos idiomas português e inglês: "atividade física", "depressão" e "idoso".

Os artigos obedeceram aos seguintes critérios de inclusão: indexação de estudos nas bases de dados escolhidas; relaçáo direta com os descritores; idiomas de publicação em português e em inglês; período de publicação compreendido entre 2010 e 2016; estudos com pesquisa de campo e artigos disponíveis on-line. Foram excluídas revisóes de literatura, dissertaçóes, teses e editoriais. A coleta de dados foi realizada seguindo os critérios de inclusão no mês de setembro de 2016.

Ao se associarem os descritores, foram encontradas 754 referências na BVS, 860 artigos no PubMed e 524 nos Periódicos Capes. Após a aplicação dos critérios de inclusão restaram 59 na BVS, 25 no PubMed e 87 nos Periódicos Capes.

$\mathrm{Na}$ primeira etapa da coleta, foi realizada a leitura dos títulos e resumos. Após essa análise, foram selecionados 24 artigos da BVS, 16 do PubMed e 20 dos Periódicos Capes. Confrontando os resultados entre as bases de dados, do total de 60 artigos, 27 se repetiram, restando assim 33 publicaçóes selecionadas para leitura integral dos textos.

$\mathrm{Na}$ segunda etapa, foi realizada a leitura integral dos artigos, com a exclusão de publicaçôes que, mesmo contemplando os descritores, não tratavam especificamente do tema proposto nesta revisáo. Assim, foram selecionados 21 artigos que compuseram a amostra final.

$\mathrm{Na}$ última etapa, os 21 artigos foram analisados a partir de conceitos predeterminados para a categorização das evidências que contemplassem respostas ao problema de pesquisa levantado. Tais conceitos foram analisados de forma descritiva e integrativa, identificando as categorias explicativas do fenômeno. 


\section{Resultados}

Houve uma produção considerável de artigos publicados referentes à temática da relação entre atividade física e depressão na população idosa entre os anos de 2013 e 2016, com 17 (81\%) publicações. Já entre os anos de 2010 e 2012 foram somente $4(19 \%)$. Esse crescimento de estudos nos últimos 4 anos demonstra sobremaneira um aumento substancial no interesse pela investigação da temática em questão.

Os artigos se caracterizam por apresentarem

$100 \%$ de abordagem metodológica quantitativa, divididos em estudos transversais, longitudinais, randomizados com grupo controle e prospectivos.

O Quadro 1 descreve o conjunto dos 21 artigos selecionados nesta revisão integrativa segundo autores, títulos, periódicos, ano de publicação e tipo de estudo.

Quadro 1: Artigos da amostra final, por ordem decrescente do ano de publicação.

\begin{tabular}{|c|c|c|c|}
\hline Autores & Título & $\begin{array}{l}\text { Periódico e ano de } \\
\text { publicaçáo }\end{array}$ & Tipo de estudo \\
\hline $\begin{array}{l}\text { Moraes H, Deslandes A, } \\
\text { Maciel-Pinheiro PT, Corrêa H, } \\
\text { Laks J }^{11}\end{array}$ & $\begin{array}{l}\text { Cortisol, DHEA, and } \\
\text { depression in the elderly: } \\
\text { the influence of physical } \\
\text { capacity }\end{array}$ & $\begin{array}{l}\text { Arquivos de } \\
\text { Neuropsiquiatria } \\
(2016)\end{array}$ & $\begin{array}{l}\text { Estudo transversal com } 32 \text { idosos depressivos e } \\
31 \text { idosos controle saudáveis submetidos a ava- } \\
\text { liaçóes de capacidade física e coleta de saliva. }\end{array}$ \\
\hline $\begin{array}{l}\text { Dotson VM, HSU FC, } \\
\text { Langaee TY, McDonough } \\
\text { CW, King AC, Cohen RA, } \\
\text { et al. }{ }^{12}\end{array}$ & $\begin{array}{l}\text { Genetic moderators of the } \\
\text { impact of physical activity } \\
\text { on depressive symptoms }\end{array}$ & $\begin{array}{l}\text { The Journal of Frailty \& } \\
\text { Aging (2016) }\end{array}$ & $\begin{array}{l}\text { Estudo randomizado controlado em } 396 \text { adul- } \\
\text { tos com idade entre } 70 \text { e } 89 \text { anos submetidos } \\
\text { à atividade física e avaliação de polimorfismos } \\
\text { em 5-HTT, BDNF e APOE; e escala de } \\
\text { depressão. }\end{array}$ \\
\hline Evans DR, Segerstrom SC ${ }^{13}$ & $\begin{array}{l}\text { Physical activity and de- } \\
\text { pressive symptoms interact } \\
\text { to predict executive func- } \\
\text { tioning among community } \\
\text { dwelling older adults }\end{array}$ & $\begin{array}{l}\text { Experimental Aging } \\
\text { Research } \\
(2015)\end{array}$ & $\begin{array}{l}\text { Estudo longitudinal com } 150 \text { idosos subme- } \\
\text { tidos à atividade física durante períodos de } \\
\text { vulnerabilidade cognitiva devido a sintomas } \\
\text { depressivos. }\end{array}$ \\
\hline $\begin{array}{l}\text { Bhamani MA, Khan MM, } \\
\text { Karim MS, Mir UM }{ }^{14}\end{array}$ & $\begin{array}{l}\text { Depression and its associa- } \\
\text { tion with functional status } \\
\text { and physical activity in the } \\
\text { elderly in Karachi, Pakistan }\end{array}$ & $\begin{array}{l}\text { Asian Journal of Psychiatry } \\
(2015)\end{array}$ & $\begin{array}{l}\text { Estudo transversal com } 953 \text { idosos submetidos } \\
\text { à avaliaçáa de atividade física e depressáo por } \\
\text { meio de aplicaçáo de questionário. }\end{array}$ \\
\hline $\begin{array}{l}\text { Roh HW, Hong CH, Lee Y, } \\
\text { Oh BH, Lee KS, Chang KJ, } \\
\text { et al. }{ }^{15}\end{array}$ & $\begin{array}{l}\text { Participation in physical, } \\
\text { social and religious } \\
\text { activity and risk of } \\
\text { depression in the elderly: } \\
\text { a community-based three- } \\
\text {-year longitudinal } \\
\text { study in Korea }\end{array}$ & $\begin{array}{l}\text { PLOS One } \\
(2015)\end{array}$ & $\begin{array}{l}\text { Estudo longitudinal avaliando } 6.647 \text { idosos } \\
\geq 60 \text { anos em relaçáo à depressáo, e às ativida- } \\
\text { des física, social e religiosa, através de escala e } \\
\text { questionários. }\end{array}$ \\
\hline $\begin{array}{l}\text { Branco JC, Jansen K, Sobrinho } \\
\text { JT, Carrapatoso S, Spessato B, } \\
\text { Carvalho J, } \\
\text { et al. }{ }^{16}\end{array}$ & $\begin{array}{l}\text { Physical benefits and } \\
\text { reduction of depressive } \\
\text { symptoms among the } \\
\text { elderly: results from the } \\
\text { Portuguese "National } \\
\text { Walking Program" }\end{array}$ & $\begin{array}{l}\text { Ciência \& Saúde Coletiva } \\
\text { (2015) }\end{array}$ & $\begin{array}{l}\text { Estudo longitudinal com } 26 \text { idosos subme- } \\
\text { tidos à avaliação física, escala de depressão e } \\
\text { questionários. }\end{array}$ \\
\hline $\begin{array}{l}\text { Belvederi Murri M, Amore M, } \\
\text { Menchetti M, Toni G, Neviani } \\
\text { F, Cerri M, } \\
\text { et al. } .^{17}\end{array}$ & $\begin{array}{l}\text { Physical exercise for late-life } \\
\text { major depression }\end{array}$ & $\begin{array}{l}\text { The British Journal of } \\
\text { Psychiatry (2015) }\end{array}$ & $\begin{array}{l}\text { Estudo cego, randomizado, com } 121 \text { pacientes } \\
\text { idosos com depressão submetidos à terapia } \\
\text { com sertralina e a atividades físicas. }\end{array}$ \\
\hline $\begin{array}{l}\text { Jimenez DE, Reynolds CF, } \\
\text { Alegría M, Harvey P, Bartels SJ }{ }^{18}\end{array}$ & $\begin{array}{l}\text { The happy older latinos are } \\
\text { active (HOLA) } \\
\text { health promotion and } \\
\text { prevention study: } \\
\text { study protocol for a pilot } \\
\text { randomized } \\
\text { controlled trial }\end{array}$ & $\begin{array}{l}\text { Trials } \\
(2015)\end{array}$ & $\begin{array}{l}\text { Estudo piloto randomizado controlado com } \\
\text { um total de } 60 \text { idosos, divididos em dois } \\
\text { grupos submetidos a duas intervençôes de } \\
\text { prevençáo de distúrbios mentais. }\end{array}$ \\
\hline
\end{tabular}


Quadro 1: Continuação.

Quadro 1: Continuação.

Yoshida Y, Iwasa H, Kumagai S, between habitual physical Suzuki T, Awata S, Yoshida $\mathrm{H}^{19}$ activity and depressive

Gonçalves AK, Griebler EM, Possamai VD, Teixeira $\mathrm{AR}^{20}$

Borges, Benedetti, Xavier, D'Orsi $^{21}$

Minghelli B,

Tomé B, Nunes C, Neves A, Simóes $\mathrm{C}^{22}$

Silva AM, Faria DS, Duarte GGM, Veiga EV, Silva PCS ${ }^{23}$

Ferreira PCS, Tavares DMS, Martins NPF, Rodrigues LR, Ferreira LA ${ }^{24}$

Figueiredo VF, Pereira LSM, Ferreira PH, Pereira AM, Amorim JSC ${ }^{25}$

Teixeira CM, Raposo JV, Fernandes HM, Brustad RJ ${ }^{26}$

Barcelos-Ferreira RB, Nakano EY, Steffens DC, Bottino $\mathrm{CMC}^{27}$

Souza DB, Serra AJ, Suzuki $\mathrm{FS}^{28}$

Huang JH, Lu YF, Cheng FC, Lee JN,

Tsai $\mathrm{LC}^{29}$

Santos KT, Fernandes MH, Reis LA, Coqueiro RS, Rocha $\mathrm{SV}^{30}$

Chang M, Jonsson PV, Snaedal The effect of midlife physical J, Bjornsson S, Saczynski JS, Aspelund T, et al..$^{31}$ mas depressivos em idosos symptoms in older people Qualidade de vida e sinto-

Psychiatry and Clinical

Neurosciences (2015) de três faixas

etárias praticantes de atividade física

Fatores associados aos sintomas depressivos em idosos: estudo EpiFloripa

Comparação dos níveis de ansiedade e depressão entre idosos ativos e sedentários

Revista de Psiquiatria

Clínica

(2013)

Avaliação da depressão e do estilo de vida de idosos hipertensos

Revista Eletrônica de Enfermagem (2013)

Características sociodemográficas e hábitos de vida de Revista Eletrônica de idosos com e sem indicativo Enfermagem (2013) de depressão

Incapacidade funcional, sintomas depressivos e dor lombar em idosos

Physical activity, depression Social indicators Research and anxiety among the (2013)

elderly

Quality of life and physical activity associated to lower prevalence of depression in community-dwelling elderly subjects from Sao Paulo

Atividade física e nível de depressão em idosas

Correlation of magnesium intake with metabolic parameters, depression and physical activity in elderly type 2 diabetes patients: a cross-sectional study

Depressive symptoms and motor performance in the elderly: a population based study activity on cognitive function among older adults: AGES-Reykjavik study

Journal of Affective

(2013)

Nutrition Journal (2012) Revista Brasileira de

Fisioterapia (2012)

The Journals of Gerontology (2010)
Estudo de coorte prospectivo com 680 idosos e diferentes escalas de depressão, submetidos a entrevista e questionário para avaliação de atividade física.

Estudo transversal com 69 idosos divididos em 3 grupos etários submetidos a escala de depressão e questionário para avaliação da qualidade de vida.

Estudo epidemiológico transversal com 1.656 idosos submetidos à escala de depressão e a um questionário de saúde, comportamental e social, incluindo atividade física.

Estudo descritivo-correlacional e transversal com 72 idosos em um grupo de 38 sedentários e outro de 34 fisicamente ativos, submetidos a um questionário sociodemográfico e escala de ansiedade e depressão.

Estudo analítico quantitativo com 102 idosos hipertensos submetidos à escala de depressão e a um questionário sociodemográfico e de atividade física.

Estudo domiciliar, analítico, transversal e observacional com 850 idosos submetidos a um questionário, incluindo avaliação de atividade física e escala de depressão.

Estudo observacional de corte transversal

Fisioterapia em Movimento com 54 idosos submetidos a questionário

(2013) sociodemográfico, de incapacidade e escala de depressão.

Estudo transversal, descritivo e correlacional com 140 idosos submetidos a escalas de atividade física, depressão e ansiedade.

Estudo transversal com 1.563 idosos submetidos a análise sociodemográfica, escalas de depressão e avaliação médica para diagnóstico.

Estudo transversal com 15 idosas com histórico de atividade física presente nos meses antecedentes, submetidas a entrevista para escala de depressão.

Estudo transversal com 210 idosos com diabetes tipo 2, submetidos a determinaçôes bioquímicas em sangue e urina, avaliação de atividade física e depressão.

Estudo transversal com 316 idosos, submetidos a avaliação sociodemográfica, incluindo atividade física e depressão.
Estudo longitudinal com 4.945 idosos com avaliação de demência e atividade física. 


\section{Discussão}

A operacionalização requerida na revisão integrativa baseou-se na categorização das evidências apresentadas nos artigos que compuseram a amostra final. Nesse sentido, após a análise foi possível realizar o agrupamento da discussão em três categorias explicativas do fenômeno, são elas: 1) relação entre atividade física e depressão em idosos; 2) fatores biológicos da atividade física e depressão em idosos; 3) atividade física em grupo e a depressão em idosos.

\section{Relação entre atividade física e depressão}

\section{em idosos}

Os resultados dos artigos analisados mostraram que a eficácia da atividade física no tratamento da depressão em idosos funciona de maneira proporcional inversa, pois o indivíduo mais ativo fisicamente apresenta menor probabilidade de desenvolver doenças e comprometer a qualidade do envelhecimento ${ }^{14,22,28}$. Outra descoberta foi a presença de baixos níveis de cortisol controlados por meio de exercício físico, o que também diminui o risco de depressão nos idosos ${ }^{11}$.

Em um estudo, os pesquisadores relacionaram a presença de depressão e ansiedade em grupos sedentários e em grupos ativos de idosos. Evidenciaram que a prática regular de atividade física pode representar um fator importante para reduzir os níveis de ansiedade e depressão na população idosa ${ }^{22}$.

Já em outro estudo, os dados mostraram que o número de idosos que não praticava atividade física foi, proporcionalmente, maior entre os que apresentavam indicativo de depressão quando comparados àqueles sem indicativo ${ }^{24}$.

Um fato importante é o tempo usado para a atividade física: foi possível constatar que idosos que praticam atividade física em uma quantidade maior que 5,2 horas por semana têm uma probabilidade $60 \%$ menor de apresentar um quadro depressivo em relaçáa àqueles que praticam menos de 2 horas por semana ${ }^{14,31}$.

Se o tempo direcionado à atividade física é um dado principal no combate à depressão, isso também pode ser afirmado em relação aos problemas funcionais atribuídos aos idosos ${ }^{25,30}$. Pesquisadores identificaram que a lombalgia, por exemplo, está associada à presença de sintomas depressivos ${ }^{25}$. Já em uma outra pesquisa, identificou-se que os sintomas da depressão estavam relacionados à perda de desempenho motor em idosos residentes na comunidade, contribuindo para o aumento das limitaçóes funcionais nos testes de desempenho motor $^{30}$. Os pesquisadores concluíram que existe uma relação inversa entre sintomas depressivos e desempenho motor $^{30}$.

A atividade física, individual ou em equipe, com um treinamento anaeróbico ou aeróbico ${ }^{17}$, tem uma eficácia surpreendente na diminuição dos sintomas depressivos e na melhora da saúde física e mental de idosos ${ }^{16}$. Logo, o exercício físico pode ser uma segura e eficiente terapia antidepressiva para essa população ${ }^{17}$.

A teoria de que a atividade física é mais significativa durante períodos de instabilidade mental pode se aplicar a uma populaçáo ampla de adultos, mais velhos do que as pesquisas anteriores mostraram ${ }^{13}$. Em um dos estudos, mesmo a bibliografia afirmando que quanto mais velho maiores são os níveis de depressão, identificou-se que ser ativo estava associado de forma mais decisiva na avaliação dos sintomas depressivos e na qualidade de vida do que estava à idade propriamente dita ${ }^{20}$.

Os aperfeiçoamentos de uma ação de promoção da saúde projetada para evitar perturbaçóes mentais comuns podem prevenir incapacitantes provenientes desse transtorno ${ }^{18}$. Uma investigação mostrou relaçáo importante entre depressão e fatores potencialmente modificáveis, reforçando o benefício de medidas preventivas para incentivo de estilo de vida saudável, atividades de lazer e da prática de exercício físico, bem como o diagnóstico e o tratamento dos sintomas depressivos ${ }^{27}$.

\section{Fatores biológicos da atividade física e depressão em idosos}

A depressão tem sido associada a alterações nos níveis de cortisol e dehidroepiandrosterona (DHEA) em idosos depressivos. As análises de uma pesquisa evidenciaram que a prática física modula a relação entre depressão e os níveis de cortisol ${ }^{11}$.

Os critérios explicativos para a associação metabólica da depressão em idosos estariam relacionados com a liberação de hormônios como a epinefrina, norepinefrina, somatotrofina, endorfina e cortisol ${ }^{11}$. Entendemse alterações no fluxo sanguíneo e no metabolismo de várias partes do cérebro correspondentes a atenção, psicomotricidade, capacidade executiva e decisão, ideias tristes e ao aprendizado emocional ${ }^{20}$.

Outra questão importante se associa à ingestão de magnésio constatada em pacientes idosos diabéticos e depressivos. Ao aumentar a ingestão de magnésio na suplementação ou na dieta, há uma melhora do organismo, da depressão e da eficiência física ${ }^{29}$. Assim, é necessária uma investigação de trabalho futura para se obter uma medida mais específica e padronizada, 
com equivalente metabólico para o controle da depressão geriátrica ${ }^{15}$.

Além de ser relacionada às alteraçóes de humor, ao pensamento suicida e a outros comportamentos, a depressão em pessoas longevas também tende a ser relacionada a alteraçóes corporais periféricas, incluindo a síndrome vegetativa e a hipercortisolemia ${ }^{26}$. Além disso, há aumento de gordura no abdômen, redução da densidade óssea e risco de evoluçáo de diabetes tipo II e hipertensão ${ }^{26}$.

\section{Atividade física em grupo e a depressão em idosos}

Uma pesquisa demonstrou que a atividade física é um importante agente de proteção contra a depressão. Além disso, é um estimulante de mudanças de hábitos saudáveis, ambientais e sociais ${ }^{19}$.

Em outro estudo, observou-se que o grupo de idosos ativos, quando comparado com o grupo de idosos sedentários, obteve menores níveis de ansiedade e/ou depressão. Esses resultados podem ter sido atribuídos não apenas pelos benefícios fisiológicos do exercício físico ${ }^{22}$, mas pela própria prática que foi realizada em grupo, contribuindo para a implementação das relaçóes sociais.

Nesse sentido, estar socialmente em atividade, principalmente, participar de confraternizaçoos ou eventos religiosos, ter relação sexual, situação clínica estável e melhores condiçôes socioeconômicas foram associados a menor predomínio de sintomatologia depressiva ${ }^{21}$.

Pesquisadores identificaram que os sintomas depressivos afetaram as pessoas longevas, como, por exemplo, na diminuição da prática de experimentar coisas prazerosas e nas dificuldades interpessoais. Tais peculiaridades, quando ausentes, estão diretamente ligadas ao aparecimento da depressão em idosos ${ }^{12}$.

$\mathrm{Na}$ população idosa, devido a uma maior vulnerabilidade às condiçóes biológicas, sociais e ambientais que reduzem a habilidade funcional, é frequente a associação com prejuízos nas funções do corpo, dificuldades no desempenho dos exercícios cotidianos e restrições na participação social do indivíduo idoso em grupos 25 .

A combinação entre a situação matrimonial e os sintomas depressivos também foi identificada em uma pesquisa, a qual demonstrou que pessoas que vivem sem companheiro têm maior prevalência de depressão. Esse dado evidencia a importância da convivência $\operatorname{cotidiana}^{23}$.

Os resultados obtidos em um estudo especificamente identificaram que a população de idosos que participavam de programas de atividade física em grupo não apresentava altos escores de depressão. Os autores então concluíram que o convívio em grupo pode ser um fator importante para alcançar bons níveis de qualidade de vida, bem como para proteger contra o desenvolvimento da depressão ${ }^{28}$.

\section{Conclusão}

A depressão é uma doença psiquiátrica cada vez mais presente na população e tem sido relacionada ao sedentarismo e isolamento social, afetando principalmente os indivíduos idosos. Assim, as evidências desta revisão identificaram que a atividade física pode ser utilizada como recurso terapêutico não medicamentoso na prevençáo e no tratamento da depressão em idosos.

Os estudos identificaram que a prática regular de atividade física melhora o condicionamento corporal, a regulação hormonal e é alternativa eficaz para o convívio social. O elo argumentativo em questáo demonstra que a prática de exercício físico em pessoas idosas tem papel fundamental na diminuição dos sintomas depressivos.

Portanto, torna-se importante destacar que para se ter um envelhecimento ativo é necessário garantir, além de saúde, a segurança, a participaçáo social e a autonomia dos idosos. São na terceira e na quarta idades, cujas características principais em relaçáo ao comportamento social são a diminuição das capacidades sensoriais e a redução da prontidão para a resposta, que a atividade física aparece de forma a contribuir para um envelhecimento com menos agravos à saúde mental, em especial a depressão.

\section{Referências}

1. Andrade LM, Sena ELS, Pinheiro GML, Meira EC, Lira LSSP. Políticas públicas para pessoas idosas no Brasil: uma revisão integrativa. Cien Saude Colet. 2013;18(12):3543-52.

2. Guimarães ACA, Scotti AV, Soares A, Fernandes S, Machado Z. Percepçáo da qualidade de vida e da finitude de adultos de meia idade e idoso praticantes e náo praticantes de atividade física. Rev Bras Geriatr Gerontol. 2012;15(4):661-70.

3. Santos GS, Cunha ICKO. Avaliação da qualidade de vida de mulheres idosas na comunidade. $\mathrm{R}$ Enferm Cent $\mathrm{O}$ Min. 2014;4(2):1135-45.

4. Campos ACV, Ferreira EF, Vargas AMD. Determinantes do envelhecimento ativo segundo a qualidade de vida e gênero. Cien Saude Colet. 2015;20(7):2221-37.

5. Lima EP, Assunção AA, Barreto SM. Prevalência de depressão em bombeiros. Cad Saúde Pública. 2015;31(4):733-43.

6. Martínez-Cengotitabengoa M, Carrascón L, O’Brien JT, Díaz-Gutiérrez MJ, Bermúdez-Ampudia C, Sanada K et al. Peripheral inflammatory parameters in late-life depression: 
a systematic review. Int J Mol Sci. 2016;17(12):1-13.

7. Rodrigues GHP, Gebara OCE, Gerbi CCS, Pierri H, Wajngarten M. Depression as a clinical determinant of dependence and low quality of life in elderly patients with cardiovascular disease. Arq Bras Cardiol. 2015;104(6):443-9.

8. Camolas J, Santos O, Moreira P, Carmo I. Promovendo mudanças comportamentais sustentáveis no controlo do peso corporal. Acta Med Port. 2014;27(1):99-107.

9. Queiroz BM, Coqueiro RS, Leal JS Neto, Borgatto AF, Barbosa AR, Fernandes MH. Inatividade física em idosos não institucionalizados: estudo de base populacional. Cien Saude Colet. 2014;19(8):3489-96.

10. Mendes KDS, Silveira RCCP, Galvão CM. Revisão integrativa: método de pesquisa para incorporação de evidências na saúde e na enfermagem. Texto Contexto Enferm. 2008;17(4):758-64.

11. Moraes H, Deslandes A, Maciel-Pinheiro PT, Corrêa H, Laks J. Cortisol, DHEA, and depression in the elderly: the influence of physical capacity. Arq Neuropsiquiatr. 2016;74(6):456-61.

12. Dotson VM, Hsu FC, Langaee TY, McDonough CW, King AC, Cohen RA, et al. Genetic moderators of the impact of physical activity on depressive symptoms. J Frailty Aging. 2016;5(1):6-14.

13. Evans DR, Segerstrom SC. Physical activity and depressive symptoms interact to predict executive functioning among community dwelling older adults. Exp Aging Res. 2015;41(5):534-45.

14. Bhamani MA, Khan MM, Karim MS, Mir MU. Depression and its association with functional status and physical activity in the elderly in Karachi, Pakistan. Asian J Psychiatr. 2015;14(1):46-51.

15. Roh HW, Hong CH, Lee Y, Oh BH, Lee KS, Chang KJ, et al. Participation in physical, social, and religious activity and risk of depression in the elderly: a community-based three-year longitudinal study in Korea. PLOS One. 2015;10(7):1-13.

16. Branco JC, Jansen K, Sobrinho JT, Carrapatoso S, Spessato B, Carvalho J, et al. Physical benefits and reduction of depressive symptoms among the elderly: results from the Portuguese "National Walking Program". Cien Saude Colet. 2015;20(3):789-95.

17. Belvederi Murri M, Amore M, Menchetti M, Toni G, Neviani F, Cerri M, et al. Physical exercise for late-life major depression. Br J Psychiatry. 2015;207(3):235-42.

18. Jimenez DE, Reynolds CF III, Alegría M, Harvey P, Bartels SJ. The happy older latinos are active (HOLA) health promotion and prevention study: study protocol for a pilot randomized controlled trial. Trials. 2015;16:579.

19. Yoshida Y, Iwasa H, Kumagai S, Suzuki T, Awata S, Yoshida $H$. Longitudinal association between habitual physical activity and depressive symptoms in older people. Psychiatry Clin Neurosci. 2015;69(11):686-92.

20. Gonçalves AK, Griebler EM, Possamai VD, Teixeira AR. Qualidade de vida e sintomas depressivos em idosos de três faixas etárias praticantes de atividade física. Kairós Gerontologia. 2014;17(3):79-94.

21. Borges LJ, Benedetti TRB, Xavier AJ, D'Orsi E. Associated factors of depressive symptoms in the elderly: EpiFloripa study. Rev Saúde Pública. 2013;47(4):1-10.

22. Minghelli B, Tomé B, Nunes C, Neves A, Simões C. Comparação dos níveis de ansiedade e depressão entre idosos ativos e sedentários. Rev Psiq Clín. 2013;40(2):71-6.

23. Silva AM, Faria DS, Duarte GGM, Veiga EV, Silva PCS. Avaliação da depressão e do estilo de vida de idosos hipertensos. Rev Eletr Enf. 2013;15(2):368-74.

24. Ferreira PCS, Tavares DMS, Martins NPF, Rodrigues LR, Ferreira LA. Características sociodemográficas e hábitos de vida de idosos com e sem indicativo de depressão. Rev Eletr Enf. 2013;15(1):197-204.

25. Figueiredo VF, Pereira LSM, Ferreira PH, Pereira AM, Amorim JSC. Incapacidade funcional, sintomas depressivos e dor lombar em idosos. Fisioter Mov. 2013;26(3):549-57.

26. Teixeira CM, Vasconcelos-Raposo J, Fernandes HM, Brustad RJ. Physical activity, depression and anxiety among the elderly. Soc Indic Res. 2013;113(1):307-18.

27. Barcelos-Ferreira R, Nakano EY, Steffens DC, Bottino CM. Quality of life and physical activity associated to lower prevalence of depression in community-dwelling elderly subjects from Sao Paulo. J Affect Disord. 2013;150(2):616-22.

28. Souza DB, Serra AJ, Suzuki FS. Atividade física e nível de depressão em idosas. R bras ci Saúde. 2012;16(1):3-6.

29. Huang JH, Lu YF, Cheng FC, Lee JN, Tsai LC. Correlation of magnesium intake with metabolic parameters, depression and physical activity in elderly type 2 diabetes patients: a cross-sectional study. Nutr J. 2012;11(41):1-10.

30. Santos KT, Fernandes MH, Reis LA, Coqueiro RS, Rocha SV. Depressive symptoms and motor performance in the elderly: a population based study. Rev Bras Fisioter. 2012;16(4):295-300.

31. Chang M, Jonsson PV, Snaedal J, Bjornsson S, Saczynski JS, Aspelund T, et al. The effect of midlife physical activity on cognitive function among older adults: AGES-Reykjavik study. J Gerontol A Biol Sci Med Sci. 2010;65(12):1369-74.

\section{Como citar este artigo:}

Mendes GAB, Carvalho MV, Silva AMTC, Almeida RJ. Relação entre atividade física e depressão em idosos: uma revisão integrativa. Rev. Aten. Saúde. 2017;15(53)110-116. 